\title{
Chemoradiation after FOLFIRINOX for borderline resectable or locally advanced pancreatic cancer
}

\author{
Brandon R. Mancini ${ }^{1 \#}$, Stacey Stein ${ }^{2 \#}$, Shane Lloyd ${ }^{3}$, Charles E. Rutter ${ }^{4}$, Edward James ${ }^{5}$, Bryan W. \\ Chang $^{6}$, Jill Lacy $^{2}$, Kimberly L. Johung ${ }^{7}$ \\ ${ }^{1}$ Department of Radiation Oncology, University of Michigan, Ann Arbor, MI, USA; ${ }^{2}$ Section of Medical Oncology, Yale University School of \\ Medicine, New Haven, CT, USA; ${ }^{3}$ Department of Radiation Oncology, Huntsman Cancer Institute, University of Utah, Salt Lake City, UT, USA; \\ ${ }^{4}$ Department of Radiation Oncology, Hartford Hospital, Hartford, CT, USA; ${ }^{5}$ Hematology/Oncology, Advocate Lutheran General Hospital, Park \\ Ridge, IL, USA; ${ }^{6}$ Department of Radiation Oncology, Torrance Memorial Medical Center, Torrance, CA, USA; ${ }^{7}$ Department of Therapeutic \\ Radiology, Yale University School of Medicine, New Haven, CT, USA \\ Contributions: (I) Conception and design: All authors; (II) Administrative support: None; (III) Provision of study materials or patients: All authors; \\ (IV) Collection and assembly of data: All authors; (V) Data analysis and interpretation: All authors; (VI) Manuscript writing: All authors; (VII) Final \\ approval of manuscript: All authors. \\ \#These authors contributed equally to this work. \\ Correspondence to: Kimberly L. Johung, MD, PhD. Department of Therapeutic Radiology, Yale University School of Medicine, P.O. Box 208040, \\ New Haven, CT 06520-8040, USA. Email: kimberly.johung@yale.edu.
}

Background: The safety and efficacy of FOLFIRINOX (FX) followed by consolidative chemoradiation (CRT) in borderline resectable (BRPC) and locally advanced pancreatic cancer (LAPC) has not been extensively studied. We sought to evaluate outcomes and toxicities of this regimen.

Methods: A retrospective review was performed of 33 patients with BRPC or LAPC treated with FX followed by CRT. Radiotherapy was directed at the primary tumor and any involved nodes $(84.8 \%$ received 50-50.4 Gy with standard fractionation and concurrent capecitabine, while $15.2 \%$ of patients received 36 Gy in 15 fractions with weekly gemcitabine). Toxicities of FX and CRT were graded using Common Terminology Criteria for Adverse Events (CTCAE v4.0), and radiographic response was evaluated using Response Evaluation Criteria in Solid Tumors (RECIST). Overall survival (OS), distant metastasisfree survival (DMFS), and local control (LC) were calculated using Kaplan-Meier analyses, and a Cox proportional hazards model was used to assess the impact of clinicopathologic factors on OS.

Results: Median follow-up was 19.9 months and patients received a median of 6.4 months of chemotherapy (range, 2.2-12.0 months). There were more T4 tumors than T3 tumors (70\% vs. 30\%). Grade $\geq 3$ toxicities were low, including fatigue (9.1\%), diarrhea (6.1\%), neuropathy (6.1\%), and dehydration (6.1\%). R0 surgical resection was achieved in 5 patients (15.2\%) after CRT. Median OS was 22.0 months (91\% at 1 year and $45 \%$ at 2 years). Median DMFS was 17.8 months (69\% at 1 year and $35 \%$ at 2 years). LC was $84 \%$ at 1 year and $55 \%$ at 2 years.

Conclusions: OS is promising with the use of FX in BRPC and LAPC, and consolidative CRT was well tolerated in this cohort. Therefore, the role of radiation after multi-agent chemotherapy should be further evaluated in prospective trials.

Keywords: Pancreatic neoplasms; radiotherapy; drug therapy

Submitted Oct 06, 2017. Accepted for publication Mar 27, 2018.

doi: 10.21037/jgo.2018.04.03

View this article at: http://dx.doi.org/10.21037/jgo.2018.04.03 


\section{Introduction}

Only $15-20 \%$ of the more than 55,000 cases of pancreatic cancer diagnosed each year in the United States are resectable at diagnosis (1), while the majority are either borderline resectable (BRPC) or locally advanced (LAPC) (30-40\%) or metastatic (40\%). Advances in chemotherapy regimens and radiation delivery are critical to improve outcomes for patients with localized yet unresectable disease, and the treatment of BRPC and LAPC pancreatic cancer continues to evolve. Current National Comprehensive Cancer Network (NCCN) guidelines for patients with LAPC recommend up-front clinical trial enrollment or combination chemotherapy for patients with adequate performance status, with chemoradiation (CRT) reserved for select patients without disease progression following a sufficient course of chemotherapy (2). In patients with BRPC, neoadjuvant chemotherapy is recommended with consideration of chemoradiation, followed by reevaluation for surgery (2).

The role of CRT in the treatment of LAPC remains controversial. Selected use of concurrent CRT in patients without early distant progression after induction chemotherapy has shown promising results in multiple retrospective and phase II studies (3-7), suggesting that this strategy identifies those patients more likely to derive a benefit from local control (LC) with chemoradiation. The LAP07 phase III trial randomized patients to CRT with capecitabine or no further therapy after 4 months of gemcitabine plus or minus erlotinib. While LC was improved with CRT (68\% vs. 54\%), there was no difference in overall survival (median OS of 15.2 months with CRT vs. 16.5 months with chemotherapy) (8). However, this trial does not reflect the current chemotherapy regimens used in advanced pancreatic cancer.

The most promising chemotherapy regimens to date have been studied in patients with metastatic disease, with practice-changing phase III studies demonstrating significant improvements in OS, progression-free survival, and response rates with FOLFIRINOX (FX) (5-fluorouracil, leucovorin, oxaliplatin, and irinotecan) compared to gemcitabine alone (9), and nab-paclitaxel plus gemcitabine compared to gemcitabine alone (10). The PRODIGE4/ ACCORD11 study randomized patients with metastatic pancreatic cancer to $\mathrm{FX}$ versus gemcitabine, and median OS was significantly prolonged with $\mathrm{FX}$ at 11.1 versus 6.8 months with gemcitabine (9). The mPACT trial demonstrated a survival benefit to gemcitabine and nab- paclitaxel compared to gemcitabine alone (median OS of 8.5 versus 6.7 months, respectively). Increasingly these regimens are being used in the treatment of LAPC (11-15). A recent meta-analysis of mostly retrospective cohort studies reported a median survival of 24.2 months for LAPC patients treated with first line FX, significantly prolonged compared to historical controls treated with gemcitabine (16). The potential benefit of local therapy in the setting of these superior systemic regimens is of interest. Therefore, the purpose of our study was to analyze the tolerability and efficacy of FX and subsequent CRT for BRPC and LAPC.

\section{Methods}

We performed an institutional review board approved retrospective review of all patients with BRPC or LAPC treated with FX followed by CRT at Smilow Cancer Hospital at Yale-New Haven Hospital between June 2010 and June 2014. Fifteen of the 33 patients reviewed were treated on protocol NCT01523457, a phase II study evaluating progression-free survival in patients with metastatic and LAPC unresectable pancreatic cancer treated with dose-attenuated modified FX (11). Designation of BRPC versus LAPC was determined following consultation with a surgical oncologist and multidisciplinary review of imaging at a dedicated gastrointestinal tumor conference, using the criteria established by the NCCN (2).

FX consisted of oxaliplatin $85 \mathrm{mg} / \mathrm{m}^{2}$ over 2 hours, followed by irinotecan 180 or $135 \mathrm{mg} / \mathrm{m}^{2}$ over 90 minutes and leucovorin $400 \mathrm{mg} / \mathrm{m}^{2}$ over 2 hours, followed by FU $300 \mathrm{mg} / \mathrm{m}^{2}$ as a bolus and $2,400 \mathrm{mg} / \mathrm{m}^{2}$ as a 46 -hour continuous infusion, with dose modifications made at the discretion of the treating physician (17). All patients received pegfilgrastim with the first cycle and subsequent cycles in the absence of severe leukocytosis. Patients were also routinely treated with prophylactic anti-emetics. The number of cycles of chemotherapy prior to concurrent CRT was determined by the treating physician, taking into account patient tolerance and disease response.

Radiotherapy was directed at the tumor via $3 \mathrm{D}$ conformal techniques or intensity modulated radiation therapy delivered with arcs. Elective lymph node basins were not irradiated. Eighty-five percent of patients received conventional fractionation to 50-50.4 Gy with capecitabine and $15 \%$ received a hypofractionated course of 36 Gy in 15 fractions with full-dose weekly gemcitabine (18).

Toxicity evaluation (history, physical examination, performance status, complete blood count, and metabolic 
Table 1 Patient and disease characteristics

\begin{tabular}{lc}
\hline Characteristic & Patients $(\mathrm{n}=33)[\%]$ \\
\hline Age (mean), year & 61 \\
Gender & \\
Male & $20[61]$ \\
Female & $13[39]$ \\
T classification & \\
T3 & $10[30]$ \\
T4 & $23[70]$ \\
N classification & \\
N0 & $25[76]$ \\
N1 & $8[24]$ \\
CA 19-9 (mean) & 1,456 \\
\hline
\end{tabular}

Table 2 Toxicities of FOLFIRINOX and chemoradiation

\begin{tabular}{lcc}
\hline Grade 3/4 event & $\begin{array}{c}\text { FOLFIRINOX, } \\
\mathrm{n}(\%)\end{array}$ & $\begin{array}{c}\text { Chemoradiation, } \\
\mathrm{n}(\%)\end{array}$ \\
\hline Hematologic & $1(3.0)$ & $1(3.0)$ \\
Neutropenia & $0(0)$ & $0(0)$ \\
Febrile neutropenia & $0(0)$ & $2(6.1)$ \\
Thrombocytopenia & & \\
Non-hematologic & $3(9.1)$ & $0(0)$ \\
Fatigue & $2(6.1)$ & $0(0)$ \\
Diarrhea & $1(3.0)$ & $0(0)$ \\
Nausea & $1(3.0)$ & $0(0)$ \\
Vomiting & $2(6.1)$ & $0(0)$ \\
Neuropathy & $2(6.1)$ & $0(0)$ \\
Dehydration & $2(6.1)$ & $0(0)$ \\
Other & &
\end{tabular}

panel) was completed at the start of each cycle of chemotherapy, as well as throughout the patient's course of concurrent CRT with weekly physician visits. The Common Terminology Criteria for Adverse Events (CTCAE v4.0) scale was utilized in grading toxicity during both induction chemotherapy and concurrent chemoradiation. All imaging studies were systematically reviewed for response using the Response Evaluation Criteria in Solid Tumors (RECIST). Routine follow-up by the treating medical and radiation oncologist included blood work and a restaging CT scan of the chest, abdomen and pelvis every three months.

Statistical analysis was performed using Stata Version 12 (StataCorp LP, College Station, TX, USA). LC, distant metastasis-free survival (DMFS), and OS were calculated using Kaplan-Meier analysis. All survival times were calculated from the time of first treatment. A Cox proportional hazards model was used to assess the impact of clinical and pathological factors on OS, including age, gender, T stage, nodal status (N1 vs. N0), and pre-treatment CA 19-9.

\section{Results}

Thirty-three patients with BRPC (10 patients) or LAPC (23 patients) treated with FX chemotherapy followed by concurrent CRT were evaluated, with a median followup of 19.9 months (range, 5.0-43.7 months). The median number of months of chemotherapy was 6.4 months (range, 2.2-12.0 months). The patient and disease characteristics are displayed in Table 1. Thirty percent of patients had T3 tumors, $70 \%$ had T4 tumors, and $24 \%$ were node positive.

Twenty-five patients (75.8\%) were treated with doseattenuated FX. The regimen of induction FX was well tolerated, with low rates of grade $\geq 3$ toxicity. The majority of patients experienced grade 1-2 fatigue, diarrhea, and/or peripheral neuropathy during induction chemotherapy. The most common acute grade $\geq 3$ toxicities during induction chemotherapy were fatigue $(9.1 \%)$, diarrhea $(6.1 \%)$, neuropathy $(6.1 \%)$, and dehydration $(6.1 \%)$ (Table 2). Common grade 1-2 toxicities during CRT included fatigue $(51.5 \%)$, nausea (30.3\%), and diarrhea (33.3\%). Only 3 patients experienced a grade $\geq 3$ toxicity during chemoradiation, and only one patient required a treatment break during chemoradiation.

Follow-up imaging was available for 31 patients. The majority of patients ( $87.1 \%$ ) had stable disease one month following consolidative chemoradiation, while 3 patients $(9.7 \%)$ had a partial response and 1 patient $(3.2 \%)$ developed progressive disease. Five patients (15.2\%, 4 of 10 with BRPC and 1 of 23 with LAPC) were converted to resectable disease and received successful R0 resections. Median OS was 22.0 months and median DMFS was 17.8 months (Table 3 and Figure 1). LC at 2 years was $55 \%$. On univariable analysis, age, gender, $\mathrm{T}$ classification, $\mathrm{N}$ classification, and initial CA 19-9 did not predict for OS (Table 4). 


\section{Discussion}

The role of CRT in the management of BRPC or LAPC remains controversial $(8,19-24)$. The randomized ECOG 4201 study showed a significant survival benefit to CRT with concurrent gemcitabine compared to gemcitabine alone, establishing a role for CRT in the current management of LAPC (24). A further survival benefit with CRT has been demonstrated in multiple retrospective and phase II studies in which CRT was employed after a period

Table 3 Kaplan-Meier estimates of local control, distant metastasisfree survival, and overall survival

\begin{tabular}{lc}
\hline Outcome & FOLFIRINOX \\
\hline Overall survival & \\
Median (months) & 22 \\
1-year & $91 \%$ \\
2-year & $45 \%$ \\
Distant metastasis-free survival & \\
Median (months) & 17.8 \\
1-year & $69 \%$ \\
2-year & $35 \%$ \\
Local control & \\
1-year & $84 \%$ \\
2-year & $55 \%$ \\
\hline
\end{tabular}

of chemotherapy to select out those patients destined for early distant failure (3-7). In contrast, the recent LAP07 randomized trial failed to demonstrate a survival benefit with the addition of chemoradiotherapy in patients with LAPC after four months of induction gemcitabine (8). With the recent advances in systemic therapy for pancreatic cancer, we sought to evaluate outcomes for BRPC and LAPC patients receiving consolidative CRT after induction FX. We report low rates of treatment-associated toxicities and a favorable median survival.

Recent studies have demonstrated the superiority of multi-agent chemotherapy regimens over gemcitabine alone in patients with metastatic disease $(9,10)$, prompting the use of FX and gemcitabine/nab-paclitaxel for LAPC disease. This single institution retrospective study suggests that CRT following FX is safe and effective in the treatment of BRPC or LAPC. The median OS of 22 months is encouraging when compared with previous studies evaluating consolidative CRT after chemotherapy regimens that are now considered outdated $(3,4,8)$. Patients in the GERCOR retrospective analysis who received induction 5-FU or gemcitabine-based chemotherapy and consolidative CRT had a median OS of 15 months (3). In addition, a retrospective study from MD Anderson Cancer Center demonstrated a median OS of 12 months for patients receiving concurrent CRT after induction gemcitabine (4). Finally, as noted above, the LAP07 trial demonstrated a median OS of 15.2 months in those patients with LAPC who went on to receive chemoradiotherapy

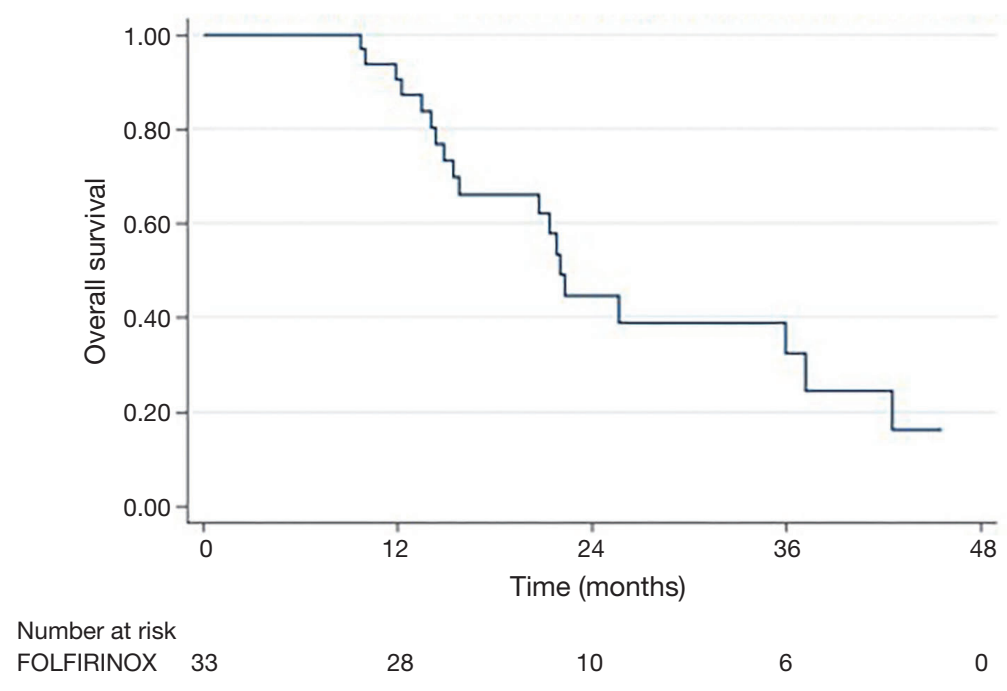

Figure 1 Overall survival for patients treated with induction FOLFIRINOX and consolidative chemoradiation. 
Table 4 Cox proportional hazard models for overall survival

\begin{tabular}{lccc}
\hline \multirow{2}{*}{ Variable } & \multicolumn{3}{c}{ Univariable analysis } \\
\cline { 2 - 4 } & HR & $95 \% \mathrm{Cl}$ & P value \\
\hline Age & 0.97 & $0.93-1.01$ & 0.19 \\
Male (vs. female) & 0.91 & $0.48-1.72$ & 0.763 \\
T4 (vs. T3) & 0.92 & $0.47-1.78$ & 0.648 \\
N1 (vs. N0) & 0.95 & $0.43-2.09$ & 0.896 \\
CA 19-9 & 1 & $1.00-1.00$ & 0.212 \\
\hline
\end{tabular}

after 4 months of chemotherapy (8). As the LAP07 trial did demonstrate improved LC and prolonged time off therapy with the addition of chemoradiation, it is possible that local therapy with radiation can impact survival outcomes in the setting of improved control of micrometastatic disease with FX.

The majority of patients in our study received attenuated doses of FX, which has been shown to reduce toxicities without compromising efficacy $(11,17)$. Our institutional prospective study of dose-attenuated FX in patients with LAPC and metastatic disease showed similar efficacy with improved tolerability compared to rates reported by Conroy et al. (9). Dose reductions in this cohort were similarly associated with improved tolerability. Moreover we demonstrate minimal toxicities during chemoradiation, with only one patient requiring a treatment break. Therefore consolidative CRT after FX may improve outcomes with minimal added morbidity, particularly with the use of IMRT which has been shown to reduce toxicities associated with combined modality therapy (25). Modified FX in sequential combination with radiation continues to be a backbone of further investigations at our institution.

The efficacy and tolerability of FX as treatment for LAPC has been reported in multiple retrospective and early phase prospective studies (11-15), including a meta-analysis in which the median OS was 24.2 months (16). In the subset of studies that reported on the use of radiation, $57 \%$ of patients received radiation, chemoradiation, or stereotactic body radiotherapy. The proportion of patients that received radiation in each study did not correlate significantly with OS, though survival outcomes for patients who received radiation were not reported. Other institutions have retrospectively reviewed their experience with $\mathrm{FX}$ followed by CRT in patients with LAPC. Faris et al. (26) reported a $23 \% \mathrm{R} 0$ resection rate following $\mathrm{FX}$ and chemoradiation. However, three of these five patients developed distant metastatic disease within five months after surgery. This patient cohort also experienced significant toxicity related to induction FX, which may be attributable to less dose reduction of chemotherapy. In a similar report, 10 patients with BRPC or LAPC went on to receive CRT after FX, and the $\mathrm{R} 0$ resection rate was $30 \%$, though grade 3 or 4 chemotherapy-related toxicities were slightly higher than our cohort (27). While these studies are limited by small cohort size, the reported $\mathrm{R} 0$ resection rates suggest an improvement in converting LAPC disease to resectable disease with this treatment regimen.

Limitations of this study include its retrospective nature, with potential for selection biases. By focusing on patients who received CRT, patients with exceptional responses to FX permitting resection were excluded from the study population. In addition, patients with early disease progression were excluded, though in our prospective institutional study, there were no patients who progressed during the initial 8 cycles of FX (11). Finally, the cohort of patients included in our analysis was small and thus standard clinicopathologic features, such as node positivity, did not correlate with outcomes.

Overall, modified FX followed by concurrent CRT is a tolerable and effective treatment regimen in BRPC and LAPC, with encouraging rates of conversion to resectable disease and survival outcomes. In the era of improved chemotherapy regimens for advanced pancreatic cancer, the role of CRT for LAPC should be re-evaluated.

\section{Acknowledgements}

None.

\section{Footnote}

Conflicts of Interest: This research was presented in part as a poster viewing presentation at the 55th Annual Meeting of the American Society for Radiation Oncology (ASTRO), September 22-25, 2013, Atlanta, GA.

Ethical Statement: The study was approved by Yale University Institutional Review Board (HIC \#1001006190). Informed consent has been waived for our study.

\section{References}

1. Siegel RL, Miller KD, Jemal A. Cancer statistics, 2017. CA Cancer J Clin 2017;67:7-30.

2. Tempero MA, Malafa MP, Al-Hawary M, et al. Pancreatic 
Adenocarcinoma, version 2.2017, NCCN Clinical Practice

Guidelines in Oncology. J Natl Compr Canc Netw

2017;15:1028-61.

3. Huguet F, Andre T, Hammel P, et al. Impact of chemoradiotherapy after disease control with chemotherapy in locally advanced pancreatic adenocarcinoma in GERCOR phase II and III studies. J Clin Oncol 2007;25:326-31.

4. Krishnan S, Rana V, Janjan NA, et al. Induction chemotherapy selects patients with locally advanced, unresectable pancreatic cancer for optimal benefit from consolidative chemoradiation therapy. Cancer 2007;110:47-55.

5. Schneider BJ, Ben-Josef E, McGinn CJ, et al. Capecitabine and radiation therapy preceded and followed by combination chemotherapy in advanced pancreatic cancer. Int J Radiat Oncol Biol Phys 2005;63:1325-30.

6. Moureau-Zabotto L, Phelip JM, Afchain P, et al. Concomitant administration of weekly oxaliplatin, fluorouracil continuous infusion, and radiotherapy after 2 months of gemcitabine and oxaliplatin induction in patients with locally advanced pancreatic cancer: a Groupe Coordinateur Multidisciplinaire en Oncologie phase II study. J Clin Oncol 2008;26:1080-5.

7. Crane CH, Varadhachary GR, Yordy JS, et al. Phase II trial of cetuximab, gemcitabine, and oxaliplatin followed by chemoradiation with cetuximab for locally advanced (T4) pancreatic adenocarcinoma: correlation of Smad4(Dpc4) immunostaining with pattern of disease progression. J Clin Oncol 2011;29:3037-43.

8. Hammel P, Huguet F, van Laethem JL, et al. Effect of Chemoradiotherapy vs Chemotherapy on Survival in Patients With Locally Advanced Pancreatic Cancer Controlled After 4 Months of Gemcitabine With or Without Erlotinib: The LAP07 Randomized Clinical Trial. JAMA 2016;315:1844-53.

9. Conroy T, Desseigne F, Ychou M, et al. FOLFIRINOX versus gemcitabine for metastatic pancreatic cancer. $\mathrm{N}$ Engl J Med 2011;364:1817-25.

10. Von Hoff DD, Ervin T, Arena FP, et al. Increased survival in pancreatic cancer with nab-paclitaxel plus gemcitabine. N Engl J Med 2013;369:1691-703.

11. Stein SM, James ES, Deng Y, et al. Final analysis of a phase II study of modified FOLFIRINOX in locally advanced and metastatic pancreatic cancer. Br J Cancer 2016;114:737-43.

12. Muranaka T, Kuwatani M, Komatsu, Y, et al. Comparison of efficacy and toxicity of FOLFIRINOX and gemcitabine with nab-paclitaxel in unresectable pancreatic cancer. J Gastrointest Oncol 2017;8:566-71.

13. Mahaseth H, Brutcher E, Kauh J, et al. Modified FOLFIRINOX regimen with improved safety and maintained efficacy in pancreatic adenocarcinoma. Pancreas 2013;42:1311-5.

14. Peddi PF, Lubner S, McWilliams R, et al. Multiinstitutional experience with FOLFIRINOX in pancreatic adenocarcinoma. JOP 2012;13:497-501.

15. Boone BA, Steve J, Krasinskas AM, et al. Outcomes with FOLFIRINOX for borderline resectable and locally unresectable pancreatic cancer. J Surg Oncol 2013;108:236-41.

16. Suker M, Beumer BR, Sadot E, et al. FOLFIRINOX for locally advanced pancreatic cancer: a systematic review and patient-level meta-analysis. Lancet Oncol 2016;17:801-10.

17. Gunturu KS, Yao X, Cong X, et al. FOLFIRINOX for locally advanced and metastatic pancreatic cancer: single institution retrospective review of efficacy and toxicity. Med Oncol 2013;30:361.

18. Small W Jr, Berlin J, Freedman GM, et al. Full-dose gemcitabine with concurrent radiation therapy in patients with nonmetastatic pancreatic cancer: a multicenter phase II trial. J Clin Oncol 2008;26:942-7.

19. Chauffert B, Mornex F, Bonnetain F, et al. Phase III trial comparing intensive induction chemoradiotherapy (60 Gy, infusional 5-FU and intermittent cisplatin) followed by maintenance gemcitabine with gemcitabine alone for locally advanced unresectable pancreatic cancer. Definitive results of the 2000-01 FFCD/SFRO study. Ann Oncol 2008;19:1592-9.

20. Sultana A, Tudur Smith C, Cunningham D, et al. Systematic review, including meta-analyses, on the management of locally advanced pancreatic cancer using radiation/combined modality therapy. Br J Cancer 2007;96:1183-90.

21. Huguet F, Girard N, Guerche CS, et al. Chemoradiotherapy in the management of locally advanced pancreatic carcinoma: a qualitative systematic review. J Clin Oncol 2009;27:2269-77.

22. Treatment of locally unresectable carcinoma of the pancreas: comparison of combined-modality therapy (chemotherapy plus radiotherapy) to chemotherapy alone. Gastrointestinal Tumor Study Group. J Natl Cancer Inst 1988;80:751-5.

23. Klaassen DJ, MacIntyre JM, Catton GE, et al. Treatment of locally unresectable cancer of the stomach and pancreas: a randomized comparison of 5 -fluorouracil alone with 
radiation plus concurrent and maintenance 5-fluorouracil-an Eastern Cooperative Oncology Group study. J Clin Oncol 1985;3:373-8.

24. Loehrer PJ Sr, Feng Y, Cardenes H, et al. Gemcitabine alone versus gemcitabine plus radiotherapy in patients with locally advanced pancreatic cancer: an Eastern Cooperative Oncology Group trial. J Clin Oncol 2011;29:4105-12.

25. Prasad S, Cambridge L, Huguet F, et al. Intensity modulated radiation therapy reduces gastrointestinal toxicity in locally advanced pancreas cancer. Pract Radiat

Cite this article as: Mancini BR, Stein S, Lloyd S, Rutter CE, James E, Chang BW, Lacy J, Johung KL. Chemoradiation after FOLFIRINOX for borderline resectable or locally advanced pancreatic cancer. J Gastrointest Oncol 2018;9(6):982-988. doi: 10.21037/jgo.2018.04.03
Oncol 2016; 6:78-85.

26. Faris JE, Blaszkowsky LS, McDermott S, et al. FOLFIRINOX in locally advanced pancreatic cancer: the Massachusetts General Hospital Cancer Center experience. Oncologist 2013;18:543-8.

27. Hosein PJ, Macintyre J, Kawamura C, et al. A retrospective study of neoadjuvant FOLFIRINOX in unresectable or borderline-resectable locally advanced pancreatic adenocarcinoma. BMC Cancer 2012;12:199. 